\title{
Reduced Peripheral Nerve Conduction Velocity is Associated with Alzheimer's Disease: A Cross-Sectional Study from China
}

\author{
Xinyi Qian $\mathbb{D}^{1,2}$, Ling Yue ${ }^{1,2}$, David Mellor $\mathbb{D}^{3}$, Nathaniel M Robbins ${ }^{4}$, Wei $\mathrm{Li}^{1,2}$, Shifu Xiao ${ }^{1,2}$ \\ 'Department of Geriatric Psychiatry, Shanghai Mental Health Center, Shanghai Jiao Tong University School of Medicine, Shanghai, People's Republic of \\ China; ${ }^{2}$ Alzheimer's Disease and Related Disorders Center, Shanghai Jiao Tong University, Shanghai, People's Republic of China; ${ }^{3}$ School of Psychology, \\ Deakin University, Melbourne, Australia; ${ }^{4}$ Department of Neurology (N.M.R.), Dartmouth-Hitchcock Medical Center, Lebanon, NH, USA
}

Correspondence: Wei Li; Shifu Xiao, Department of Geriatric Psychiatry, Shanghai Mental Health Center, Shanghai Jiao Tong University School of Medicine, 600 Wan Ping Nan Road, Shanghai, 200030, People’s Republic of China, Tel +86 21-34773758; +86 21-64387250, Email 822203867@qq.com; xiaoshifu@msn.com

Purpose: Elderly individuals with degenerative diseases of the central nervous system are more likely to develop peripheral neuropathy; however, research is limited as to whether the decline in peripheral nerve conduction can be used as a biomarker of Alzheimer's disease (AD).

Patients and Methods: This study enrolled 74 patients with mild cognitive impairment (MCI), 21 with AD, and 82 healthy elderly individuals. All participants underwent a peripheral nerve conduction and neuropsychological evaluation. Nicolet EDX was used to assess peripheral nerve conduction in the limbs and comparisons were made between the three cognitive groups. Furthermore, the relationship between peripheral nerve conduction and cognitive function was investigated.

Results: A ladder-shaped difference was found in the median $(p<0.001)$ and common peroneal $(p<0.001)$ motor nerve velocity, with the control group $>$ MCI group $>$ AD group, even after controlling for variables. The median motor nerve amplitude in the AD group was lower than that in the control group $(\mathrm{P}=0.017)$. After controlling for age, sex, education, and height, the median motor nerve velocity was positively correlated with the Montreal Cognitive Assessment $(r=0.196, p=0.015)$, and the common peroneal motor nerve velocity was positively correlated with verbal fluency task-idioms $(r=0.184, p=0.026)$. The median (AUC: 0.777 , $p<$ 0.001 ) and common peroneal motor nerve velocities (AUC: $0.862 ; \mathrm{p}<0.001$ ) were significantly associated with the diagnosis of AD. The accuracy rate of these two motor nerve velocities to predict $\mathrm{AD}$ was $51.5 \%$.

Conclusion: Our study found that peripheral motor nerve velocity may correlate with early cognitive impairment in AD. However, the accuracy of different cognitive classifications and the value of early diagnosis are not ideal when peripheral motor nerve velocity is used alone. Whether peripheral nerve function can be used as a marker for early diagnosis of AD needs further clarification but provides a new possibility for the future of biomarker research.

Keywords: Alzheimer's disease, mild cognitive impairment, peripheral motor nerve velocity, biomarker, cognition

\section{Introduction}

Alzheimer's disease (AD) is a central neurodegenerative disease characterized by progressive cognitive impairment, specific types of neurons, and synaptic loss. ${ }^{1}$ In Shanghai, $33.5 \%$ of the elderly suffer from cognitive impairment and dementia due to central nervous degenerative diseases, of which $\mathrm{AD}$ accounts for more than $60 \%{ }^{2}$ There is mounting evidence that central nervous system (CNS) degeneration occurs as early as years to decades before the onset of cognitive symptoms, which will eventually promote the transformation of individuals to dementia. ${ }^{3}$ Unfortunately, the current treatment is unsatisfactory following the development of dementia, which is a devastating blow to patients and caregivers. As such, there is a pressing need for an effective and safe method to help identify cognitive impairment in the early stage. ${ }^{4,5}$ 
Mild cognitive impairment (MCI) is a type of cognitive impairment syndrome that occurs between that of healthy aging and dementia. MCI has no effect on the abilities of daily life and is not sufficient to be diagnosed with dementia. ${ }^{6}$ Studies have shown that $10-15 \%$ of MCI patients develop dementia, of which the majority of cases present as AD, every year, ${ }^{7}$ while the proportion of healthy elderly developing dementia is only around $1-2 \%$ per year. ${ }^{8}$ However, not all MCI patients develop dementia. Through certain interventions and training, individuals with MCI can maintain their cognitive state or even revert to normal. ${ }^{9,10}$ Therefore, MCI is an important stage in the early identification and treatment of cognitive dysfunction and prevention of MCI progressing to dementia. Although current guidelines support a relatively simple method to determine reversible or structural causes, in recent years, the field has witnessed the rapid development of complex imaging, biomarkers, and genetic research. ${ }^{11}$ The $\mathrm{AT}(\mathrm{N})$ biomarker system, in which the three letters separately represent biomarkers related to $\mathrm{A} \beta$ pathology, tau protein pathology, and neurodegeneration or neuronal injury, was proposed in 2018 for the diagnosis and further research of $\mathrm{AD} .{ }^{12}$ Guidelines for the appropriate use of $\mathrm{A} \beta$-PET and the ratio of cerebrospinal fluid (CSF) $\mathrm{A} \beta 42$ to $\mathrm{A} \beta 40$ (A $342 / \mathrm{A} \beta 40)$ in clinical practice have already been established. ${ }^{13,14}$ They can be used for the diagnosis of AD and to accurately predict the development of $\mathrm{AD}$ dementia in patients with $\mathrm{MCI}$ with high accuracy. ${ }^{15}$ Clinical research has shown that tau-positron emission tomography (PET) has an even higher specificity than that of A $\beta$-PET and CSF A $\beta 42 / A \beta 40$ in distinguishing $\mathrm{AD}$ dementia from other neurodegenerative diseases and is a better prognostic marker of $\mathrm{AD}$ than $\mathrm{A} \beta$-PET and structural magnetic resonance imaging. ${ }^{15}$ However, owing to the price and complexity of sample collection, CSF and brain imaging biomarkers cannot be widely used for early screening in primary care. Therefore, more operable peripheral biomarkers such as plasma protein levels and intestinal microbiota abundance are being widely studied. Of these, the use of peripheral nerve conduction velocity to identify cognitive impairment may a viable new research direction.

As an extension of the CNS, the peripheral nervous system (PNS), which consists of the autonomic nervous system (ANS) and somatic nervous system (SNS), plays an important role in the transmission of nerve impulses between the CNS and other organs. It has been preliminarily found that the incidence of both ANS and SNS degeneration in patients with CNS degenerative diseases is higher than that in age-matched healthy group. ${ }^{16}$ Moreover, motor disorders such as gait and postural changes, motor retardation, and even stiffness and tremor occur before the onset of significant cognitive symptoms in $\mathrm{AD}$ patients, ${ }^{17}$ suggesting a correlation between central and peripheral neuropathy. However, few studies have focused on motor and sensory nerve functions of the limbs, especially in the human body. An animal experiment showed that in the Tg2576 mouse model of $\mathrm{AD}$, had a reduced number of sciatic nerve fibers and showed obvious hindlimb extension reflex defects and abnormal tremors. ${ }^{18}$ In the human body, hyperphosphorylation of tau protein, a characteristic pathological change in $\mathrm{AD}$, was found in the sciatica of $\mathrm{AD}$ patients, ${ }^{19}$ suggesting that there may be structural and functional changes in the PNS of AD patients.

Neurophysiological measurements are the most commonly used tools for diagnosing peripheral neuropathy. ${ }^{20,21}$ Nerve conduction detection makes use of the excitable and conductive characteristics of the peripheral nerve to record the response potential produced by the nerve impulse, which is a convenient, noninvasive, inexpensive, and reproducible examination to detect somatic nerve function. ${ }^{22,23}$ The main detection parameters include nerve action potential latency, amplitude, and conduction velocity. Abnormalities in these parameters reflect the loss of nerve conduction fibers or axonal degeneration, the core pathologic progress of a neurodegenerative disease. ${ }^{24}$ Considering this potential pathological link, it is worth exploring whether peripheral somatic nerve conductive function is associated with $\mathrm{AD}$.

In this study, we hypothesized that peripheral somatic neuroconduction decreases during $\mathrm{MCI}$ and $\mathrm{AD}$, and aimed to explore the internal relationship between peripheral somatic neuroconduction and cognitive function.

\section{Materials and Methods}

\section{Participants}

A total of 177 subjects participated in this study, including 82 healthy controls (HC), 74 patients with MCI, and 21 patients with AD. The MCI and HC groups were selected from the Shanghai Brain Health Cohort Study, while patients with $\mathrm{AD}$ were selected from the geriatric psychiatric ward of the Shanghai Mental Health Center. All participants signed 
an informed consent form at the start of the study, and ethical approval was obtained from the Shanghai Mental Health Center.

All subjects were aged between 55 and 85 years, regardless of sex. AD and MCI were diagnosed according to the diagnostic criteria for AD developed by the National Institute on Aging-Alzheimer's Association (NIA-AA) ${ }^{25}$ and Peterson's MCI criteria, ${ }^{26}$ respectively. Patients with a history of organic brain diseases, brain trauma, head surgery, depression, alcoholism, drug abuse, neurological disease affecting peripheral nerves (eg, CIDP, toxins, and paraneoplastic syndrome), cognitive impairment caused by diseases other than $\mathrm{AD}$, and positive signs on physical examination of the nervous system (ie, touch, temperature, pain and proprioceptive examination of limbs, and tendon reflex of extremities) were excluded.

Ethical approval was obtained from the Ethics Committee of the Shanghai Mental Health Center and all participants provided signed informed consent before the study.

All the experimental procedures were carried out in strict accordance with the approved procedures.

\section{Demographic and Neuropsychological Tests}

The subjects' general demographic characteristics (ie, age, sex, total years of education, height, and handedness), daily living data (ie, smoker, drinker), and disease-related information (ie, hypertension and diabetes) were obtained by means of self-report. A series of neuropsychological tests, such as the Montreal Cognitive Assessment (MoCA), ${ }^{27}$ Rey Auditory-Verbal Learning Test (RAVLT), ${ }^{28}$ trail making test (TMT), ${ }^{29}$ digit span(DS), ${ }^{30}$ and verbal fluency task (VFT) ${ }^{31}$ were used to assess overall and domain-specific cognitive function. Considering the degree of cooperation among inpatients with $\mathrm{AD}$, subjects in the $\mathrm{AD}$ group only completed the MoCA.

\section{Nerve Conduction Detection}

A nerve conduction study of the median nerve (sensory and motor nerves), sural sensory nerve, and common peroneal motor nerve was performed using NicoletTM EDX (Natus Neurology Incorporated. Germany) in accordance with the standard procedure. ${ }^{32,33}$ The latency, amplitude, and velocity of nerve conduction were used as the main parameters. The latency and amplitude of median motor nerve conduction were measured at surface projection of median nerve at wrist and elbow (recorded as "wrist" and "elbow"), while the latency and amplitude of common peroneal motor nerve conduction were measured below the capitulum of fibula (recorded as "knee") and at the anterior malleolus (recorded as "ankle"). There was only one measurement point for each or the other main parameters.

All subjects measured their right limbs in a unified manner, unless the subjects were left-handed. If the right limb had a history of fracture, carpal tunnel syndrome, or other discomfort, the contralateral limb was measured instead. All nerve conduction tests were performed in a quiet, independent examination room at a temperature of $22-24^{\circ} \mathrm{C}$. If the extremities were at a low temperature, the test was performed after keeping warm indoors.

\section{Statistical Analysis}

SPSS 22.0 (IBM Corporation, Armonk, NY, USA) was used for data entry and analysis. The Kolmogorov-Smirnov test was used to test the normal distribution of each variable, and a p-value $>0.05$ was considered as being in accordance with the positive distribution. According to the distribution characteristics of variables, the independent sample $t$-test or Mann Whitney $U$-test was used for quantitative variables, and the chi-square test or Fisher's exact test was used for categorical variables. Differences between the groups were compared using one-way analysis of variance and then analyzed using Bonferroni correction. Kruskal-Wallis test was used if the variance was not uniform. Spearman correlation analysis was used to compare the results of nerve conduction detection with neuropsychological test scores. The Benjamin-Hochberg method (BH method) was used to correct the correlation results. If univariate analysis showed a significant correlation, we further compared whether there was a linear relationship after controlling for gender, age, and years of education. Finally, Fisher discriminant analysis and receiver operating characteristic curves were used to analyze whether the results of the nerve conduction test with statistical differences among groups could correctly classify the cognitive level and diagnose the disease. 


\section{Results}

\section{Demographic and Neuropsychological Tests}

There was no difference in sex between the three groups, but there were differences in age, education level, height, and the total MOCA score. Specifically, education level $(p<0.01)$ and total MOCA score $(p<0.001)$ in the NC group were higher than those in the MCI group, and the total MOCA score in the MCI group was higher $(\mathrm{p}<0.001)$ than that in the AD group. However, the heights of the NC and MCI groups were lower than that of the AD group $(\mathrm{p}<0.05)($ Table 1$)$.

\section{Nerve Conduction Detection}

All subjects completed upper limb detection. In the NC group, five subjects did not complete the sural sensory nerve conduction test because they could not tolerate the continuous superimposed current. Two subjects in the NC group, three subjects in the $\mathrm{MCI}$ group, and two subjects in the AD group could not elicit the sural sensory nerve conduction results. Two subjects in the NC group, two subjects in the MCI group, and one subject in the AD group refused to perform lowerlimb detection because of discomfort in upper-limb detection. One subject in the NC group and two subjects in the AD group rejected the lower limb detection owing to inconvenient clothing. In the MCI group, one subject failed to complete lower-limb detection due to lower-limb paralysis. One patient in the AD group did not complete the common peroneal motor nerve conduction test. Three subjects in NC group, three subjects in MCI group and two subjects in AD group measured left limbs because of the history of right limb fracture.

There was a statistically significant difference in the amplitude (wrist) and velocity of median motor nerve conduction, as well as the latency (knee) and velocity of common peroneal motor nerve conduction among the three groups. The amplitude (wrist) of median motor nerve conduction in the AD group was smaller than that in the other two groups (AD group vs MCI group: $\mathrm{p}=0.037$; $\mathrm{AD}$ group vs $\mathrm{NC}$ group: $\mathrm{p}=0.002$ ). The motor nerve velocities of the median nerve and common peroneal nerve decreased step-by-step in the three groups ( $\mathrm{NC}$ group $>\mathrm{MCI}$ group $>\mathrm{AD}$ group). There was no significant difference in the pairwise comparison of the latency (knee) of common peroneal motor nerve conduction among the three groups (Table 2).

Since age, sex, and height were found to be the influencing factors of peripheral nerve conduction, ${ }^{34}$ we made further analysis by controlling for age, sex, and height. The median motor nerve and common peroneal motor nerve velocities were different in pairwise comparisons among the three groups. Similarly, a difference in median motor nerve amplitude (wrist) still existed between the $\mathrm{AD}$ and $\mathrm{NC}$ groups ( $\mathrm{p}=0.017$ ) (Figure 1).

\section{Correlation Between Nerve Conduction and Neuropsychological Tests}

After using the BH method for the False Discovery Rate (FDR) correction of Spearman correlation analysis, both the velocity of the median motor nerve and the common peroneal motor nerve were found to be significantly correlated with the total MoCA score $(\mathrm{p}<0.001)$. The common peroneal motor nerve velocity was correlated with the idiom part $(\mathrm{p}=0.01)$ and vegetable part of the VFT ( $\mathrm{p}=0.013)$ and the 20 minute-delayed recall of AVLT ( $\mathrm{p}<0.001)$ (Table 3$)$. After controlling for related variables, including age, sex, education level, height, and geriatric depression scale (GDS) score, the median motor nerve velocity was still correlated with the total MOCA score $(\mathrm{r}=0.196, \mathrm{p}=0.015)$, and the common peroneal nerve motor conduction velocity was still correlated with the idiom part of the VFT $(r=0.184, p=0.026)$ (Figure 2).

Table I Comparison of Demographic Data and MoCA Score Among the Three Groups

\begin{tabular}{|c|c|c|c|c|c|}
\hline & MCI Group $(n=74)$ & NC Group $(n=82)$ & AD Group $(n=2 I)$ & $\mathrm{F} / \mathrm{H} / \chi^{2}$ Value & p-value \\
\hline Age\# & $70.36 \pm 7.3$ & $68.04 \pm 6.27$ & $73.33 \pm 9.06$ & 6.692 & $0.035 *$ \\
\hline Gender (male, \%) & $22(29.73 \%)$ & $23(28.05 \%)$ & $7(33.3 \%)$ & 0.233 & 0.894 \\
\hline Education level (year) ${ }^{\mathrm{a}}$ & $10.05 \pm 3.58$ & $11.45 \pm 2.57$ & $10.38 \pm 4.13$ & 3.799 & $0.024 *$ \\
\hline Height (meter) ${ }^{b, c}$ & $1.61 \pm 0.08$ & $1.61 \pm 0.07$ & $1.66 \pm 0.07$ & 4.157 & $0.017^{*}$ \\
\hline Total score of $M o C A^{a, b, c}$ & $|8.3| \pm 3.29$ & $24.24 \pm 2.97$ & $4.48 \pm 4.13$ & $3|4.86|$ & $<0.001 * *$ \\
\hline
\end{tabular}

Notes: Continuous data are presented as mean \pm standard deviation. ${ }^{*} \mathrm{p}<0.05 ;{ }^{*} \mathrm{p}<0.00 \mathrm{I}$. ${ }^{\mathrm{a} P o s t}$ analysis showed difference between $\mathrm{NC}$ group and $\mathrm{MCl}$ group. ${ }^{\mathrm{b}} \mathrm{Post}$




Table 2 Comparison of Peripheral Nerve Conduction Test Among the Three Groups

\begin{tabular}{|c|c|c|c|c|c|c|}
\hline & & MCI Group $(n=74)$ & NC Group $(n=82)$ & AD Group $(n=21)$ & F/H Value & p-value \\
\hline \multirow[t]{3}{*}{ Median Sensory Nerve } & Lat & $2.88 \pm 0.65$ & $2.83 \pm 0.64$ & $2.82 \pm 0.46$ & 0.128 & 0.880 \\
\hline & Amp & $5.82 \pm 3.34$ & $5.18 \pm 4.72$ & $4.01 \pm 1.75$ & 1.766 & 0.174 \\
\hline & Vel & $53.86 \pm 9.44$ & $53.62 \pm 9.20$ & $52.22 \pm 7.77$ & 0.269 & 0.765 \\
\hline \multirow[t]{3}{*}{ Sural Sensory Nerve } & Lat & $3.14 \pm 1.01$ & $3.24 \pm 1.11$ & $3.74 \pm 1.06$ & 2.058 & 0.131 \\
\hline & Amp & $3.21 \pm 3.36$ & $4.39 \pm 6.16$ & $3.29 \pm 2.89$ & 1.132 & 0.325 \\
\hline & Vel & $41.34 \pm 11.82$ & $40.98 \pm 12.88$ & $38.46 \pm 15.69$ & 0.336 & 0.716 \\
\hline \multirow[t]{5}{*}{ Median Motor Nerve } & Lat (wrist) & $3.40 \pm 0.82$ & $3.25 \pm 0.63$ & $3.44 \pm 0.57$ & 1.103 & 0.334 \\
\hline & Lat (elbow) & $7.65 \pm 1.11$ & $7.32 \pm 0.85$ & $7.61 \pm 0.70$ & 2.428 & 0.091 \\
\hline & Amp (wrist) ${ }^{b, c}$ & $6.82 \pm 2.69$ & $7.46 \pm 2.57$ & $5.17 \pm 2.81$ & 6.328 & $0.002^{* *}$ \\
\hline & Amp (elbow) & $6.04 \pm 2.70$ & $6.6 \mathrm{I} \pm 2.52$ & $5.31 \pm 2.92$ & 2.312 & 0.102 \\
\hline & $V_{e l}{ }^{a, b}, c$ & $54.51 \pm 4.18$ & $56.36 \pm 4.72$ & $50.43 \pm 4.41$ & 15.189 & $<0.001 * *$ \\
\hline \multirow[t]{5}{*}{ Common Peroneal Motor Nerve } & Lat (ankle) & $4.49 \pm 1.59$ & $4.72 \pm 1.82$ & $5.13 \pm 1.62$ & 1.013 & 0.365 \\
\hline & Lat (knee) & $12.01 \pm 2.19$ & $11.96 \pm 2.23$ & $|3.37 \pm 1.9|$ & 3.093 & $0.048 *$ \\
\hline & Amp (ankle) & $0.76 \pm 0.74$ & $0.69 \pm 0.67$ & $0.73 \pm 0.91$ & 0.193 & 0.824 \\
\hline & Amp (knee) & $0.79 \pm 0.91$ & $0.68 \pm 0.5 \mathrm{I}$ & $0.65 \pm 0.57$ & 0.519 & 0.596 \\
\hline & $\operatorname{Vel}^{\#, a, b, c}$ & $47.04 \pm 5.90$ & $49.38 \pm 5.63$ & $41.18 \pm 2.17$ & 28.736 & $<0.00 I^{* *}$ \\
\hline
\end{tabular}

Notes: Continuous data are presented as mean \pm standard deviation. ${ }^{*} \mathrm{p}<0.05 ;{ }^{*} \mathrm{p}<0.00 \mathrm{I}$. ${ }^{\text {a }}$ Post analysis showed difference between NC group and MCl group. ${ }^{\mathrm{b}}$ Post analysis showed difference between NC group and AD group. ${ }^{C}$ Post

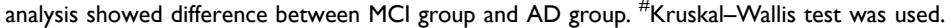



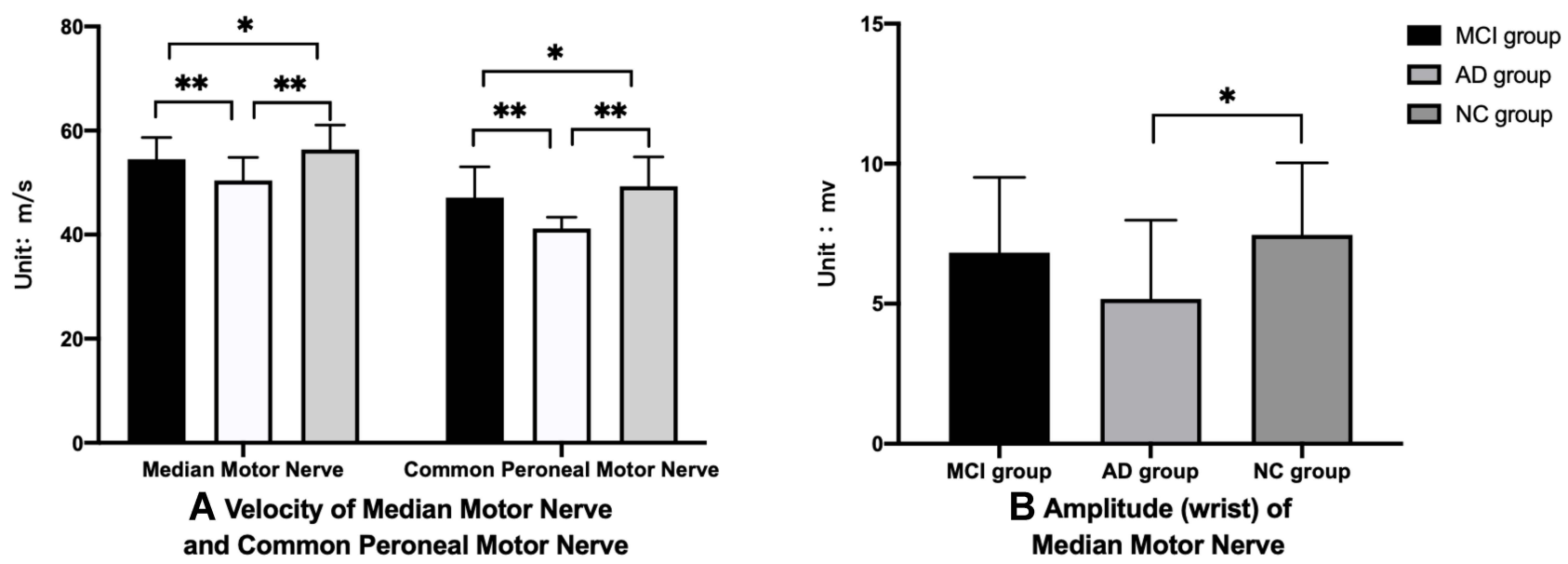

Figure I Comparison of peripheral motor nerve velocity and median motor nerve amplitude among the three groups after controlling variables. * $<<0.05$; $* * p<0.00$ I. $(\mathbf{A})$ The median motor nerve and common peroneal motor nerve velocities were different in pairwise comparisons among the three groups. (B) The median motor nerve amplitude (wrist) was different between the AD and NC groups.

Further logistic regression was used to determine causality in the correlation. When establishing the model, variables known to be risk factors for cognitive decline were included, including age, gender, and years of education. Slow velocities of both the median (OR: $0.81, \mathrm{p}=0.01$ ) and common motor nerve (OR: $0.74, \mathrm{p}<0.001$ ) were risk factors for $\mathrm{AD}$, but not for MCI (Table 4).

\section{Classification Analysis Results}

Using the ROC curve, we found no value for the above two motor nerve velocities in the diagnosis of MCI. In the diagnosis of $\mathrm{AD}$, both the median (AUC: $0.777,95 \% \mathrm{Cl}: 0.677-0.877, \mathrm{p}<0.001$ ) and common peroneal motor nerve velocities (AUC, 0.862; 95\% Cl: 0.804-0.920, $\mathrm{p}<0.001$ ) showed valuable results (Figure 3). The cutoff for the median motor velocity was $56.20 \mathrm{~m} / \mathrm{s}$, and that for the common peroneal motor nerve velocity was $43.60 \mathrm{~m} / \mathrm{s}$.

Fisher's discriminant analysis was used to analyze the grouping accuracy of the median and common peroneal motor nerve velocities at different cognitive levels. Subjects without common peroneal motor nerve conduction data were excluded from the discriminant analysis. Compared with the original group, 48 cases in $\mathrm{NC}$ group were correctly grouped, with an accuracy rate of $60.8 \%$. In MCI group, 24 patients were correctly grouped, with an accuracy rate of $33.8 \%$. In AD group, 14 cases were correctly grouped, with an accuracy rate of $82.4 \%$. The total accuracy was $51.5 \%$ (Table 5).

Table 3 Correlation Between Median and Common Peroneal Motor Nerve Velocity and Neuropsychological Test

\begin{tabular}{|l|l|l|l|l|}
\hline \multirow{2}{*}{} & \multicolumn{2}{|l|}{ Velocity of Median Motor Nerve } & \multicolumn{2}{l|}{ Velocity of Common Peroneal Motor Nerve } \\
\cline { 2 - 5 } & Correlation Coefficient $\mathbf{r}$ & $\mathbf{p}$ value & Correlation Coefficient r & p value \\
\hline MoCA & $0.364^{* *}$ & $<0.00 I^{* *}$ & $0.400^{* *}$ & $<0.00 I^{* *}$ \\
TMT-A & -0.110 & 0.189 & -0.109 & 0.206 \\
TMT-B & -0.085 & 0.291 & $-0.175^{*}$ & 0.057 \\
AVLT-Delayed recall $(5 \mathrm{~min})$ & 0.157 & 0.167 & $0.172^{*}$ & 0.05 \\
AVLT-Delayed recall $(20 \mathrm{~min})$ & $0.19 I^{*}$ & 0.155 & $0.313^{* *}$ & $<0.00 I^{* *}$ \\
DS (Normal order) & 0.137 & 0.174 & 0.105 & 0.199 \\
DS (Reverse order) & 0.135 & 0.133 & 0.119 & 0.185 \\
VFT (Vegetables) & 0.146 & 0.17 & $0.234^{* *}$ & $0.013^{*}$ \\
VFT (Fruits) & 0.124 & 0.154 & $0.177^{*}$ & 0.06 \\
VFT (Idioms) & 0.138 & 0.147 & $0.236^{* *}$ & $0.0 I^{*}$ \\
\hline
\end{tabular}

Notes: ${ }^{*} p<0.05 ;{ }^{* *} p<0.001$. 

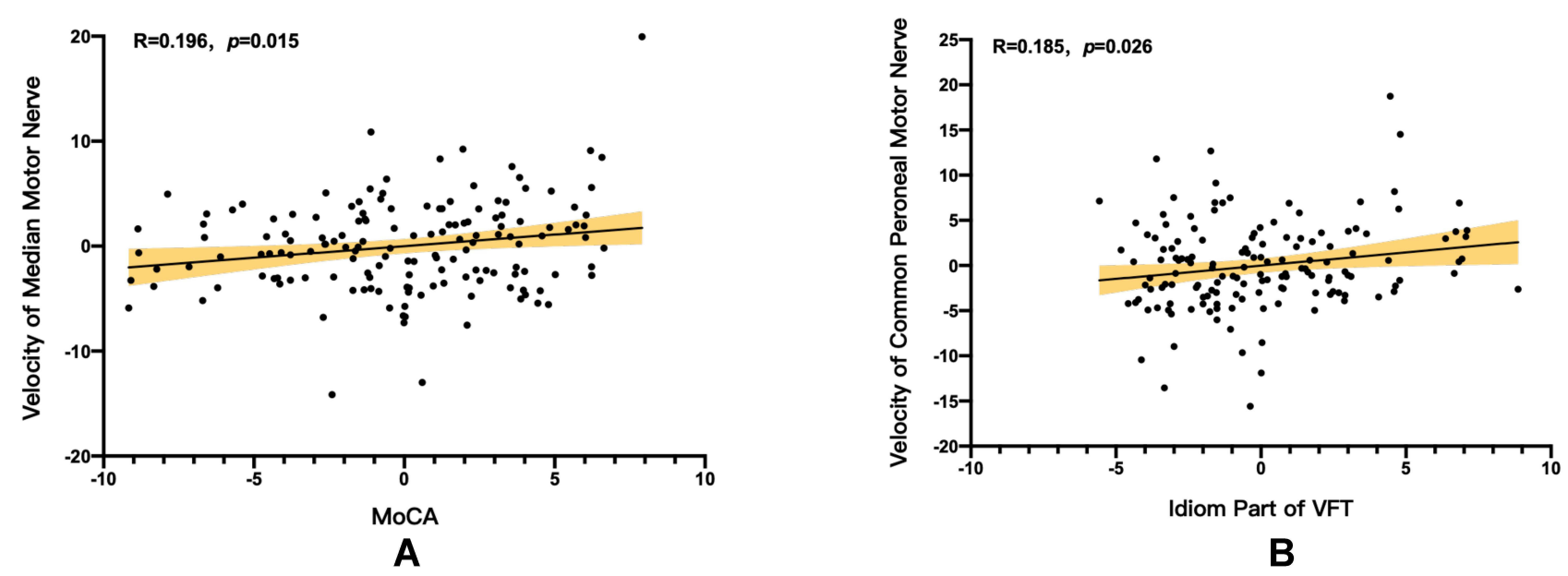

Figure 2 Correlation between median and common peroneal motor nerve velocity and neuropsychological test after controlling variables including age, gender, educational level, height and GDS score. (A)Median motor nerve velocity was correlated with the total MoCA score $(r=0.196, p=0.015)$. (B) Common peroneal motor nerve velocity was correlated with the idiom part of VFT $(r=0.185, p=0.026)$.

The MoCA was further combined with the above two motor nerve velocities to predict different cognitive levels. Compared with the original group, 68 cases in NC group were correctly grouped, with an accuracy rate of $86.1 \%$. In total, 56 cases in MCI group were correctly grouped, with an accuracy rate of $78.9 \%$. Fifteen patients in AD group were correctly grouped, with an accuracy rate of $88.2 \%$. The total accuracy was $83.2 \%$ (Table 6 ).

\section{Discussion}

Owing to the progress of aging, $\mathrm{AD}$ has become a health and social problem requiring research attention. A large number of studies have focused on AD markers in the central and peripheral systems and have achieved some positive results. However, after considering a series of factors, such as operability, acceptability, economic factors, sensitivity, and specificity, the early screening of high-risk people who may progress to AD still depends on neuropsychological tests. It is necessary to find a fast, easy, and objective method that can be used for early screening in community hospitals or memory clinics.

Our study creatively evaluated the peripheral nerve conduction of the elderly with different cognitive levels and found a positive correlation between peripheral motor nerve conduction velocity and cognitive function, suggesting that peripheral motor nerve conduction function may further decline with the decline of cognitive function.

By detecting and analyzing the conductive function of peripheral nerves in the elderly with different cognitive levels, we found a ladder-shaped difference in the median and common peroneal motor nerve velocity (NC group $>$ MCI group $>\mathrm{AD}$ group), suggesting that the motor-related fast conduction fibers of the peripheral nerve were damaged and motor conduction decreased in patients with cognitive impairment, especially in patients with AD. The median motor nerve amplitude (wrist), but not the median motor nerve amplitude (elbow), was larger in the NC group than in the AD group, suggesting that $\mathrm{AD}$ patients may have a decrease in the number of axons and/or axonal injury of median nerve motorrelated fibers. In addition, the motor nerve velocities of the median nerve and common peroneal nerve were positively correlated with the MoCA and the idiom part of the VFT, respectively, suggesting that the peripheral motor nerve

Table 4 Logistic Regression Analysis Among MCl, AD and Controls After Multivariable Adjustment

\begin{tabular}{|l|c|c|c|c|}
\hline \multirow{2}{*}{} & \multicolumn{2}{|c|}{ MCI vs NC } & \multicolumn{2}{|c|}{ AD vs NC } \\
\cline { 2 - 5 } & OR (95\% Cl) & p-value & OR (95\% CI) & p-value \\
\hline Median Motor Nerve Velocity & $0.93(0.86-1.02)$ & 0.125 & $0.81(0.69-0.95)$ & $0.01 *$ \\
Common Peroneal Motor Nerve Velocity & $0.95(0.89-1.02)$ & 0.148 & $0.74(0.63-0.86)$ & $<0.001 * *$ \\
\hline
\end{tabular}

Notes: The reference category used was NC. The model was adjusted for sex, age, and years of education. ${ }^{*} p<0.05 ; * * p<0.001$. 

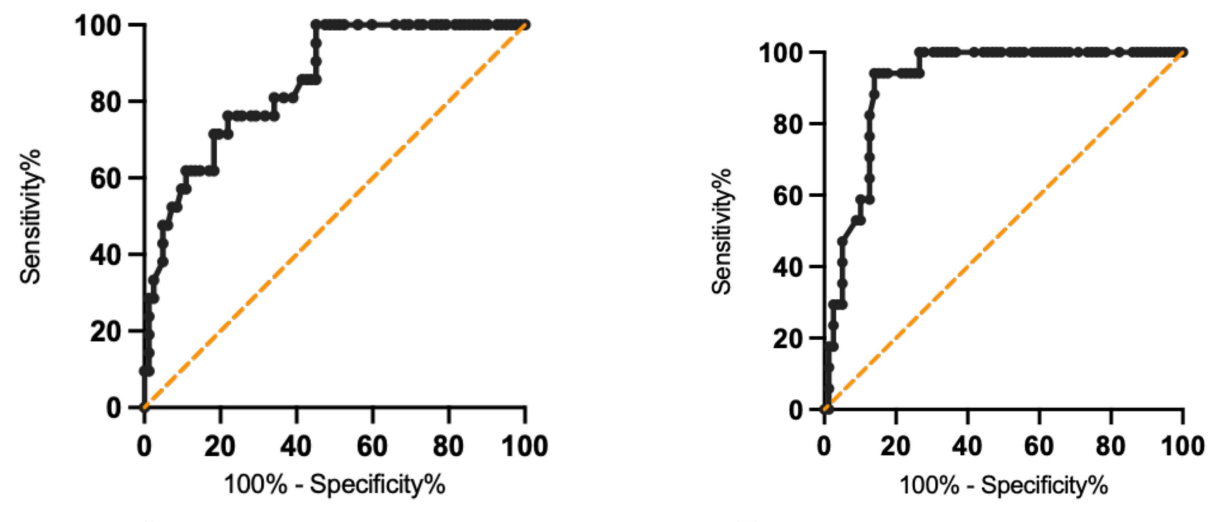

A Median Motor Nerve Vel

\section{B Common Peroneal Motor Nerve Vel}

Figure 3 The ROC curve of the median and common peroneal motor nerve velocities in the diagnosis of AD. (A) The AUC of median motor nerve velocity was 0.777 (95\% Cl: $0.677-0.877, p<0.001)$. (B) The AUC of common peroneal motor nerve velocity was 0.862 (95\% Cl: $0.804-0.920, p<0.001$ ).

conductive function may further decline with the decline of cognitive function. In terms of peripheral sensory nerve conductive function, no obvious abnormalities were found in patients with $\mathrm{MCI}$ and $\mathrm{AD}$ in our study.

\section{Peripheral Motor Nerve Conduction and Cognitive Function}

Our findings on peripheral motor nerve conduction can explain, to some extent, the emergence of dyskinesia before the typical cognitive symptoms of $\mathrm{AD} .{ }^{17}$ The occurrence of gait abnormalities and motor retardation may be related to abnormalities in the peripheral motor nerve conduction. Basic research may indirectly explain these results. Holzer et al found elevated levels of hyperphosphorylated tau protein in the sciatic nerves of AD patients, ${ }^{19}$ suggesting that tau hyperphosphorylation may occur in the peripheral nervous system. The role of the tau proteins in neurons is to participate in the formation of microtubules and regulate their stability, which is related to the repair and regeneration of peripheral nerves. ${ }^{35}$ The ability of tau proteins to stabilize microtubules depends on its isomer and the degree of phosphorylation. The binding ability of hyperphosphorylated tau protein to the microtubules was significantly reduced. ${ }^{36}$ In contrast to the central nerves, hyperphosphorylated tau proteins in the peripheral nerves are not necessarily accompanied by the formation of paired helices, ${ }^{19}$ which can lead to the production of neurofibrillary tangles, a characteristic pathological

Table 5 Prediction of Peripheral Motor Nerve Velocity in Different Cognitive Groups

\begin{tabular}{|l|c|c|c|c|}
\hline \multirow{2}{*}{ Original Group } & \multicolumn{3}{|c|}{ Forecast Group (Correct Grouping Accuracy, \%) } \\
\cline { 2 - 5 } & NC Group & MCI Group & AD Group & Total \\
\hline NC group & $48(60.8 \%)$ & $18(22.8 \%)$ & $13(16.5 \%)$ & 79 \\
MCl group & $27(38 \%)$ & $24(33.8 \%)$ & $20(28.2 \%)$ & 71 \\
AD group & $0(0 \%)$ & $3(17.6 \%)$ & $1482.4 \%)$ & 17 \\
\hline
\end{tabular}

Note: $51.5 \%$ of the original grouped cases were correctly classified.

Table 6 Prediction of MoCA and Peripheral Motor Nerve Velocity in Different Cognitive Groups

\begin{tabular}{|l|c|c|c|c|}
\hline \multirow{2}{*}{ Original Group } & \multicolumn{3}{|c|}{ Forecast Group (Correct Grouping Accuracy, \%) } \\
\cline { 2 - 5 } & NC Group & MCI Group & AD Group & Total \\
\hline NC group & $68(86.1 \%)$ & $11(13.9 \%)$ & $0(0 \%)$ & 79 \\
MCl group & $13(18.3 \%)$ & $56(78.9 \%)$ & $2(2.8 \%)$ & 71 \\
AD group & $0(0 \%)$ & $2(11.8 \%)$ & $1582.4 \%)$ & 17 \\
\hline
\end{tabular}

Note: $83.2 \%$ of the original grouped cases were correctly classified. 
change in $\mathrm{AD}$. However, tau proteins can still affect axon transport and myelin regulate on in a variety of ways, ${ }^{37,38}$ resulting in damage to the structure and function of peripheral nerves. Yin et $\mathrm{al}^{39}$ found that Tau 58/4 mice, a transgenic tau mouse that is usually used to study tauopathies including $\mathrm{AD}$, had motor function damage and the appearance of hyperphosphorylated tau proteins at the age of 3 months, which deteriorated with age. Furthermore, 12-month-old Tau 58/4 mice showed loss of large axonal fibers and hypomyelination in the sciatic nerve, which proved that hyperphosphorylated tau protein can cause motor dysfunction by causing peripheral nerve axonal degeneration and myelin abnormalities. Lopes et $\mathrm{al}^{40}$ studied another transgenic tau mouse, Tau-/- mice, and found that compared with mildtype mice and adult Tau-/- mice, the exercise coordination, endurance, and muscle strength of elderly Tau-/- mice were significantly reduced. The ultrastructure of the sciatic nerve showed myelinated fiber degeneration and conduction property damage in the elderly Tau-/- mice. In addition, the structure and regeneration of large-diameter myelin were reduced in both adult and elderly Tau-/- mice, suggesting that dyskinesia in mice may be caused by changes in sciatic nerve structure and function, and this pathological change was found to be more related to the loss of tau protein with normal function than to the increase in age.

However, the above animal studies on the possible pathological mechanism can only simulate a feature or symptom in the complex pathological process of AD, which is not exactly the same as the pathological mechanism of AD patients. In contrast to rodents, regeneration of nerve axons and myelin in humans is very slow. Degeneration and regeneration occur simultaneously in the dynamic process of neuropathology. Injury to any link may directly affect the final result of myelination and axon regeneration.

Unfortunately, an extremely limited clinical study on peripheral nerve conductive function in AD has put forward conclusions contrary to this study. A domestic study found no significant difference in median and tibial motor nerve conduction between the AD group and the age-sex-matched control group or between the AD group with dyskinesia and the AD group without dyskinesia. The conductive function of the superior neurons of the peripheral motor nerve and anterior horn neurons of the spinal cord in patients with $\mathrm{AD}$ was not significantly damaged. Therefore, this study concluded that the motor conduction pathway in patients with $\mathrm{AD}$ is not significantly damaged and that motor disorders are more related to dysfunction of the cholinergic system. An animal experiment ${ }^{41}$ confirmed the conclusions of the study. It was found that $5 \mathrm{xFAD}$ mice, a type of transgenic AD mouse model, showed damage in multiple fields of motor function compared with wild-type mice. However, isometric tension recordings of the soleus muscle did not reveal any defects in the ability of peripheral motor nerves to drive muscle output in 5xFAD mice, suggesting that motor impairments may not be due to peripheral nerve dysfunction. Although these two studies were inconsistent with our results, the nerves selected in the above clinical study were not exactly the same as those in our study, and the sample size was limited. The above animal experiment used indirect evidence to speculate on peripheral nerve function, instead of directly observing whether there were changes in the structure or detecting peripheral nerve conductive function in $5 x F A D$ mice.

Including our study, there is no direct or powerful evidence to prove or deny the relationship between dyskinesia in $\mathrm{AD}$ and peripheral motor nerve function. However, demyelination and regeneration of the distal peripheral nerve are common age-related events, and elderly individuals are more prone to peripheral nerve demyelination and injury. Therefore, even if tau protein-related pathology does not directly damage the myelin of peripheral nerves, the negative effects on myelin regeneration and axon transport caused by tauopathy may be the reason for the decline in peripheral nerve motor function in patients with $\mathrm{AD}$.

Although our study found differences in peripheral motor nerve conductive function among the elderly with different cognitive levels, the motor nerve conduction velocity of the median and common peroneal nerves was only valuable in the diagnosis of AD. The accuracy of using the above two motor nerve conductive velocities as a group to classify cognitive level was acceptable only for the AD group but not ideal for the MCI and NC groups. The overall classification accuracy is not ideal. At present, the peripheral motor nerve conductive function is not sufficient to be used alone as a marker for the early recognition of $\mathrm{AD}$. In the future, we may combine peripheral nerve conduction velocity with other peripheral biomarkers, such as plasma P-tau, plasma apolipoprotein, and exosomes, and explore their application potential in the early screening of AD. Further research is necessary to explore the relationship between peripheral motor nerves and cognitive function. 


\section{Peripheral Sensory Nerve Conduction and Cognitive Function}

In terms of sensory function in AD patients, most studies focus on olfactory, visual, and auditory impairments. Few studies have focused on limb sensation. A clinical study found a lower degree and frequency of pain in patients with AD than in cognitively healthy elderly, ${ }^{42}$ suggesting that there may be changes in peripheral nerve sensory function in patients with AD. Our study did not support this conclusion and found no peripheral sensory nerve conduction impairment in patients with MCI or AD. Sotiropoulos et $\mathrm{al}^{43}$ found that compared with $\mathrm{Tau}+/+$ mice, age-and sexmatched wild-type mice had reduced sensitivity to acute nociceptive behavior, but the change in sensory nerve conductive function was not obvious. Jensen-Dahm et $\mathrm{al}^{44}$ supported our results. They detected the contact thermal painevoked potential of extremities in patients with mild to moderate AD and the control group, and found no difference between the two groups, as well as the score of pain intensity, suggesting that the response to external pain stimulation and peripheral sensory nerve conductive function in $\mathrm{AD}$ patients were preserved.

We acknowledge that our study has several limitations. First, this was a cross-sectional study, and it was impossible to establish causality between peripheral nerve conduction velocity and cognitive function. Second, the relatively small sample size reduces the reliability of the study. Third, the AD patients in the ward were taking cognitive drugs, which may have influenced the results.

\section{Conclusion}

The conduction velocity of peripheral nerves slows down with a decline in cognitive function. The overall accuracy of using peripheral motor nerve conductive velocity alone to classify the cognitive level is not ideal, suggesting that whether peripheral nerve function can be used as a marker for early diagnosis of AD still needs to be further clarified, However, it provides a new possibility for future biomarker research.

\section{Ethics Approval and Informed Consent}

The study was conducted in accordance with the guidelines of the Declaration of Helsinki and was approved by the Ethics Committee of the Shanghai Mental Health Center.

\section{Acknowledgments}

Thank all subjects and community hospital staff for their help in the study.

\section{Funding}

This study was supported by grants from the Clinical Research Center Project of Shanghai Mental Health Center (CRC2017ZD02), Shanghai Brain Health Foundation (SHBHF2016001), Shanghai Clinical Research Center for Mental Health (19MC1911100), Cultivation of Multidisciplinary Interdisciplinary Project in Shanghai Jiaotong University (YG2019QNA10), curriculum reform of Medical College of Shanghai Jiaotong University, and Feixiang Program of Shanghai Mental Health Center (2020-FX-03). This project was also funded by the Shanghai Elderly Brain Health Cohort Institute, Chinese Academy of Sciences (XDA12040101), Shanghai Clinical Research Center for Mental Health (SCRC-MH, 19MC1911100), National Natural Science Foundation of China (82001123), Clinical Research Plan of SHDC (SHDC2020CR1038B), and Shanghai Science and Technology Committee (20Y11906800).

\section{Disclosure}

The authors declare no conflicts of interest.

\section{References}

1. Scheltens P, Blennow K, Breteler MM, et al. Alzheimer's disease. Lancet. 2016;388(10043):505-517. doi:10.1016/S0140-6736(15)01124-1

2. Xiao S, Lewis M, Mellor D, et al. The China longitudinal ageing study: overview of the demographic, psychosocial and cognitive data of the Shanghai sample. J Ment Health. 2016;25(2):131-136. doi:10.3109/09638237.2015.1124385

3. Kempuraj D, Thangavel R, Natteru PA, et al. Neuroinflammation induces neurodegeneration. J Neurol Neurosurg Spine. $2016 ; 1: 1$.

4. Tan CC, Yu JT, Tan L. Biomarkers for preclinical Alzheimer's disease. J Alzheimers Dis. 2014;42(4):1051-1069. doi:10.3233/JAD-140843 
5. Carneiro P, Morais S, Do Carmo Pereira M. Biosensors on the road to early diagnostic and surveillance of Alzheimer's disease. Talanta. 2020;211:120700. doi:10.1016/j.talanta.2019.120700

6. Sanford AM. Mild cognitive impairment. Clin Geriatr Med. 2017;33(3):325-337. doi:10.1016/j.cger.2017.02.005

7. Hänninen T, Hallikainen M, Tuomainen S, Vanhanen M, Soininen H. Prevalence of mild cognitive impairment: a population-based study in elderly subjects. Acta Neurol Scand. 2002;106(3):148-154. doi:10.1034/j.1600-0404.2002.01225.x

8. Bischkopf J, Busse A, Angermeyer MC. Mild cognitive impairment-a review of prevalence, incidence and outcome according to current approaches. Acta Psychiatr Scand. 2002;106(6):403-414. doi:10.1034/j.1600-0447.2002.01417.x

9. Cui MY, Lin Y, Sheng JY, Zhang X, Cui RJ. Exercise intervention associated with cognitive improvement in Alzheimer's Disease. Neural Plast. 2018;2018:9234105. doi:10.1155/2018/9234105

10. Bredesen DE, Amos EC, Canick J, et al. Reversal of cognitive decline in Alzheimer's disease. Aging. 2016;8(6):1250-1258. doi:10.18632/ aging.100981

11. Ganesh A, Genesh P, Adil MM, Varma M, Smith EE, Bartolini L. Practice current: how do you manage mild cognitive impairment? Neurology. 2020;10(4):362-370. doi:10.1212/CPJ.0000000000000890

12. Jack CR, Bennett DA, Blennow K, et al. NIA-AA research framework: toward a biological definition of Alzheimer's disease. Alzheimers Dement. 2018;4(14):535-562. doi:10.1016/j.jalz.2018.02.018

13. Johnson KA, Minoshima S, Bohnen NI, et al. Appropriate use criteria for amyloid PET: a report of the amyloid imaging task force, the society of nuclear medicine and molecular imaging, and the Alzheimer's association. Alzheimers Dement. 2013;9(1):E1-E16. doi:10.1016/j.jalz.2013.01.002

14. Shaw LM, Arias J, Blennow K, et al. Appropriate use criteria for lumbar puncture and cerebrospinal fluid testing in the diagnosis of Alzheimer's disease. Alzheimers Dement. 2018;14(11):1505-1521. doi:10.1016/j.jalz.2018.07.220

15. Hansson O. Biomarkers for neurodegenerative diseases. Nat Med. 2021;27(6):954-963. doi:10.1038/s41591-021-01382-x

16. Fotiou DF, Stergiou V, Tsiptsios D, Lithari C, Nakou M, Karlovasitou A. Cholinergic deficiency in Alzheimer's and Parkinson's disease: evaluation with pupillometry. Int J Psychophysiol. 2009;73(2):143-149. doi:10.1016/j.ijpsycho.2009.01.011

17. Wilson RS, Bennett DA, Gilley DW, Beckett LA, Schneider JA, Evans DA. Progression of parkinsonian signs in Alzheimer's disease. Neurology. 2000;54(6):1284-1289. doi:10.1212/WNL.54.6.1284

18. Seo JS, Leem YH, Lee KW, Kim SW, Lee JK, Han PL. Severe motor neuron degeneration in the spinal cord of the Tg2576 mouse model of Alzheimer disease. J Alzheimers Dis. 2010;21(1):263-276. doi:10.3233/JAD-2010-091528

19. Holzer M, Holzapfel HP, Krohn K, Gertz HJ, Arendt T. Alterations in content and phosphorylation state of cytoskeletal proteins in the sciatic nerve during ageing and in Alzheimer's disease. J Neural Transm. 1999;106(7-8):743-755. doi:10.1007/s007020050195

20. Griffith KA, Dorsey SG, Renn CL, et al. Correspondence between neurophysiological and clinical measurements of chemotherapy-induced peripheral neuropathy: secondary analysis of data from the CI-PeriNomS study. J Peripher Nerv Syst. 2014;19(2):127-135. doi:10.1111/jns5.12064

21. Kandula T, Farrar MA, Cohn RJ, et al. Chemotherapy-induced peripheral neuropathy in long-term survivors of childhood cancer: clinical, neurophysiological, functional, and patient-reported outcomes. JAMA Neurol. 2018;75(8):980-988. doi:10.1001/jamaneurol.2018.0963

22. Thabit MN, Sedky A, Sayed MA, Baddary HM, Mohamed M. Double-shock stimulation of the superficial radial nerve compared with standard medial plantar nerve conduction in the early detection of asymptomatic diabetic neuropathy: a Pilot Study. J Clin Neurophysiol. 2018;35 (6):474-480. doi:10.1097/WNP.0000000000000522

23. Wells MD, Meyer AP, Emley M, Kong X, Sanchez R, Gozani SN. Detection of lumbosacral nerve root compression with a novel composite nerve conduction measurement. Spine. 2002;27(24):2811-2819. doi:10.1097/00007632-200212150-00016

24. Weber GA. Nerve conduction studies and their clinical applications. Clin Podiatr Med Surg. 1990;7(1):151-178.

25. McKhann GM, Knopman DS, Chertkow H, et al. The diagnosis of dementia due to Alzheimer's disease: recommendations from the national institute on aging-Alzheimer's association workgroups on diagnostic guidelines for Alzheimer's disease. Alzheimers Dement. $2011 ; 7(3)$ :263-269. doi:10.1016/j.jalz.2011.03.005

26. Petersen RC, Smith GE, Waring SC, Ivnik RJ, Tangalos EG, Kokmen E. Mild cognitive impairment: clinical characterization and outcome. Arch Neurol. 1999;56(3):303-308. doi:10.1001/archneur.56.3.303

27. Ciesielska N, Sokołowski R, Mazur E, Podhorecka M, Polak-Szabela A, Kędziora-Kornatowska K. Is the Montreal Cognitive Assessment (MoCA) test better suited than the Mini-Mental State Examination (MMSE) in mild cognitive impairment (MCI) detection among people aged over 60? Meta-analysis. Psychiatr Pol. 2016;50(5):1039-1052. doi:10.12740/PP/45368

28. Fard EK, Keelor JL, Bagheban AA, Keith RW. Comparison of the Rey Auditory Verbal Learning Test (RAVLT) and digit test among typically achieving and gifted students. Iran J Child Neurol. 2016;10(2):26-37.

29. Llinàs-Reglà J, Vilalta-Franch J, López-Pousa S, Calvó-Perxas L, Torrents Rodas D, Garre-Olmo J. The Trail Making Test. Assessment. 2017;24 (2):183-196. doi:10.1177/1073191115602552

30. Woods DL, Kishiyamaa MM, Lund EW, et al. Improving digit span assessment of short-term verbal memory. J Clin Exp Neuropsychol. 2011;33 (1):101-111. doi:10.1080/13803395.2010.493149

31. Piskunowicz M, Bieliński M, Zgliński A, Borkowska A. [Verbal fluency tests-application in neuropsychological assessment]. Psychiatr Pol. 2013;47(3):475-485. Polish.

32. Omejec G, Podnar S. Utility of nerve conduction studies and ultrasonography in ulnar neuropathies at the elbow of different severity. Clin Neurophysiol. 2020;131(7):1672-1677. doi:10.1016/j.clinph.2020.02.019

33. Sonoo M, Menkes DL, Bland JDP, Burke D. Nerve conduction studies and EMG in carpal tunnel syndrome: do they add value? Clin Neurophysiol Pract. 2018;3:78-88. doi:10.1016/j.cnp.2018.02.005

34. Awang MS, Abdullah JM, Abdullah MR, et al. Nerve conduction study among healthy Malays. The influence of age, height and body mass index on median, ulnar, common peroneal and sural nerves. Malays J Med Sci. 2006;13(2):19-23.

35. Zha GB, Shen M, Gu XS, Yi S. Changes in microtubule-associated protein tau during peripheral nerve injury and regeneration. Neural Regen Res. 2016;11(9):1506-1511. doi:10.4103/1673-5374.191227

36. Bramblett GT, Goedert M, Jakes R, Merrick SE, Trojanowski JQ, Lee VM. Abnormal tau phosphorylation at Ser396 in Alzheimer's disease recapitulates development and contributes to reduced microtubule binding. Neuron. 1993;10(6):1089-1099. doi:10.1016/0896-6273(93)90057-X

37. LaPointe NE, Morfini G, Pigino G, et al. The amino terminus of tau inhibits kinesin-dependent axonal transport: implications for filament toxicity. J Neurosci Res. 2009;87(2):440-451. doi:10.1002/jnr.21850 
38. Klein C, Kramer EM, Cardine AM, Schraven B, Brandt R, Trotter J. Process outgrowth of oligodendrocytes is promoted by interaction of fyn kinase with the cytoskeletal protein tau. $J$ Neurosci. 2002;22(3):698-707. doi:10.1523/JNEUROSCI.22-03-00698.2002

39. Yin Z, Valkenburg F, Hornix BE, et al. Progressive motor deficit is mediated by the denervation of neuromuscular junctions and axonal degeneration in transgenic mice expressing mutant (P301S) tau protein. J Alzheimers Dis. 2017;60(s1):S41-S57. doi:10.3233/JAD-161206

40. Lopes S, Lopes A, Pinto V, et al. Absence of tau triggers age-dependent sciatic nerve morphofunctional deficits and motor impairment. Aging Cell. 2016;15(2):208-216. doi:10.1111/acel.12391

41. O'Leary TP, Robertson A, Chipman PH, Rafuse VF, Brown RE. Motor function deficits in the 12 month-old female 5xFAD mouse model of Alzheimer's disease. Behav Brain Res. 2018;337:256-263. doi:10.1016/j.bbr.2017.09.009

42. Scherder E, Bouma A, Borkent M, Rahman O. Alzheimer patients report less pain intensity and pain affect than non-demented elderly. Psychiatry. 1999;62(3):265-272. doi:10.1080/00332747.1999.11024871

43. Sotiropoulos I, Lopes AT, Pinto V, et al. Selective impact of tau loss on nociceptive primary afferents and pain sensation. Exp Neurol. 2014;261:486-493. doi:10.1016/j.expneurol.2014.07.008

44. Jensen-Dahm C, Madsen CS, Waldemar G, et al. Contact Heat Evoked Potentials (CHEPs) in patients with mild-moderate Alzheimer's disease and matched control-a Pilot Study. Pain Med. 2016;17(4):675-684. doi:10.1093/pm/pnv012

\section{Publish your work in this journal}

Neuropsychiatric Disease and Treatment is an international, peer-reviewed journal of clinical therapeutics and pharmacology focusing on concise rapid reporting of clinical or pre-clinical studies on a range of neuropsychiatric and neurological disorders. This journal is indexed on PubMed Central, the 'PsycINFO' database and CAS, and is the official journal of The International Neuropsychiatric Association (INA). The manuscript management system is completely online and includes a very quick and fair peer-review system, which is all easy to use. Visit http://www.dovepress.com/testimonials.php to read real quotes from published authors.

Submit your manuscript here: https://www.dovepress.com/neuropsychiatric-disease-and-treatment-journal 